\title{
La imagen de las políticas en los medios de comunicación*
}

\author{
Esther Forgas Berdet
}

Universidad Rovira i Virgili

\section{Presentación}

El presente artículo trata de preguntarse por los rasgos definitorios de un tipo de tratamiento periodístico: el que la prensa hispana de uno y otro continente da a las mujeres que intervienen en la vida pública, especialmente a las presidentas en activo y a candidatas a la presidencia de los países occidentales. Podríamos analizar, en este sentido, el tratamiento que recibió y recibe Cristina Fernández de Kirchner, el de Michelle Bachelet y el de muchas otras mujeres dedicadas a la política, como es el caso de la que nos ocupa en este trabajo: la excandidata francesa al Elíseo, Ségolène Royal.

En esta primera aproximación nos dedicaremos a señalar los aspectos más relevantes del enfoque desigual y, por tanto, sesgado que la prensa dispensa a estos personajes públicos, frente sus compañeros varones, y lo ejemplificaremos mediante el tratamiento dado a la política gala en la pasada campaña electoral, tomando como referencia las noticias y artículos de opinión aparecidos en cinco periódicos: tres españoles (La Vanguardia, El Mundo y $A B C$ ) y dos hispanoamericanos (La Nación de Buenos Aires y El Universal de México), a lo largo de los cuatro primeros meses de 2007.

*Este artículo fue presentado en forma de comunicación en el XXXVIII Simposio de la Sociedad Española de Lingüística, celebrado en Logroño del 17 al 20 de diciembre de 2007. 
Los puntos que abordaremos en este estudio son los siguientes:

1. El nombre de las mujeres: nombres de pila, apelativos, diminutivos, motes y apodos.

2. El aspecto físico: el atractivo, la seducción, los engaños femeninos y la ropa.

3. La condición femenina: el hecho de ser mujer.

4. La condición civil: el papel de hija, compañera (o esposa) y madre.

\section{Cómo nombran los periódicos a las mujeres: el nombre de pila}

Sabido es que a las mujeres se nos llama con mayor frecuencia por el nombre de pila que a los hombres, que las mujeres solemos presentarnos por nuestro nombre con más asiduidad que nuestros congéneres y que, en general, no nos molesta ni nos humilla el trato coloquial; es más, en la mayoría de los casos lo propiciamos. Cualquiera de nosotras puede comprobar, independientemente de dónde ejerza su profesión, que a sus compañeros se los suele llamar por el apellido, mientras que a ella se la conoce generalmente por el nombre. Hasta aquí nada que objetar, pues somos principalmente las mujeres las que deseamos ser tratadas de manera distendida, con toda naturalidad, por el nombre con que se nos conoce en nuestra familia y entre nuestras amistades. La mujer, por lo general, mantiene mucho menos las distancias, es más cercana y empática en sus relaciones sociales, y el uso del nombre de pila habitual es una manera de expresar esta característica.

Sin embargo, una cosa es lo que las mujeres aceptamos, y aun propiciamos, en nuestras relaciones personales y laborales y otra muy distinta es que los medios de comunicación se tomen la libertad de repetirlo en sus rotativos, usando una familiaridad que no creemos que sea en absoluto querida ni auspiciada por la mujer que se dedica profesionalmente a una actividad como la política, en la que está en continua y desigual competición con sus compañeros varones, a los que nadie, si no es en muy contadas ocasiones, se atrevería a llamar por su nombre de pila en un artículo de opinión o en una noticia periodística de carácter político. ${ }^{2}$ Ahí interviene el sesgo discriminador, cuando las mujeres que ocupan altos cargos de responsabilidad

\footnotetext{
${ }^{2}$ No es prerrogativa exclusiva de Ségolène, ni tampoco de los diarios hispanos. Baste como prueba un extenso artículo del periodista estadounidense Dick Morris reproducido en La Nación del 2 de enero, cuyo título "Hillary, como Cristina" es ya
} 
pública dejan de ser la Sra. Aguirre, la candidata Clinton o las presidentas Bachelet o Fernández de Kirchner, para pasar a ser, simplemente, Esperanza, Michelle, Hillary o Cristina. Indudablemente, es cierto que el nombre de pila acerca a las políticas a su electorado, y de buen seguro que muchas veces este acercamiento es propiciado por ellas mismas, pero no es menos cierto que en ciertos momentos, ante confrontaciones electorales o frente al debate serio y en profundidad, el nombre de pila rebaja la categoría del personaje representado. El mensaje subliminal de un texto que nos explica que Esperanza (por Esperanza Aguirre, por tomar un ejemplo patrio) se ha enfrentado en un duro debate con el señor Ruiz Gallardón (jamás con Alberto) no puede dejarnos indiferentes.

En ocasiones el nombre lo es todo, el nombre es la cosa, nos guste o no a los lingüistas, y la prensa escrita que crea conciencia, que educa la opinión, conforma así el imaginario colectivo. Pues bien, en el caso de la política gala, nos hallamos ante un ejemplo flagrante. En todos los periódicos consultados Ségolène (cuando no, simplemente Sego) es un nombre pila profusamente empleado para nombrar a la candidata a la presidencia de Francia, frente a, por supuesto, el absolutamente inexistente Nicolas (por Sarkozy), ${ }^{3}$ del que no hemos encontrado un solo ejemplo. No pensemos que se trata de un caso aislado, debido a la originalidad del nombre de la candidata francesa, pues el ejemplo anteriormente citado de Esperanza Aguirre echaría por tierra tal ingenua hipótesis.

Tan evidente es lo que apuntamos que incluso en el ciberespacio se han creado diferencias durante la campaña: mientras que el sitio web del can-

toda una declaración de principios. En él se puede leer que "Hillary, por ejemplo, disfruta de una amplia confianza pública en los Estados Unidos, de que Cristina puede tener una ventaja en la percepción de que podrá lidiar en forma efectiva con las finanzas internacionales". Si bien hemos de agradecer la paridad con que el periodista trata el matrimonio del exmandatario estadounidense ("su imagen [la de Cristina] está mucho más entrelazada con la del Presidente que la de Hillary con Bill"), no ocurre lo mismo con quien en aquel momento era presidente de Argentina: "Si el presidente Kirchner ha trabajado para eliminar la corrupción en el nivel federal de gobierno, Cristina debería sacar ventaja de la integridad que deviene del estereotipo femenino".

${ }^{3}$ Solamente hemos encontrado una referencia a Nicolas, pero no es creación del periodista, sino reproducción de las palabras de terceras personas: "Intimados a «votar por Nicolas o quedarse sin su banca en las legislativas de junio próximo», 19 de los 28 diputados centristas rindieron el estandarte. La Nación, 29 de abril. 
didato era "sarkozy.fr", el de la candidata era, cómo no, algo distinto: "segosphere.net".

Veamos un pequeño resumen (sin pretensiones de exhaustividad) de los porcentajes obtenidos en la prensa hispana consultada, al contabilizar los distintos apelativos:

\begin{tabular}{|l|l|l|l|l|}
\cline { 2 - 5 } \multicolumn{1}{c|}{} & $\begin{array}{l}\text { Ségolène } \\
\text { Royal }\end{array}$ & Ségolène & Royal & Sego \\
\hline La Vanguardia & $54,9 \%$ & $36,6 \%$ & $4,2 \%$ & $4,2 \%$ \\
\hline ABC & $32,2 \%$ & $26,7 \%$ & $35,4 \%$ & $0 \%$ \\
\hline El Mundo & $41,1 \%$ & $55,8 \%$ & $2,9 \%$ & $0 \%$ \\
\hline La Nación & $28 \%$ & $65 \%$ & $2,2 \%$ & $4,4 \%$ \\
\hline El Universal & $34,2 \%$ & $54,6 \%$ & $9,8 \%$ & $1,3 \%$ \\
\hline
\end{tabular}

Destacaremos de este cuadro los porcentajes pequeños, pero significativos, de las Ségolène y Sego aparecidas en La Vanguardia, El Mundo y El Universal, frente a la absoluta inexistencia de nombres de pila masculinos (Nicolas, Jacques o Lionel, por ejemplo), así como la exagerada proporción de apelativos coloquiales ( $35 \%$ ) con que $A B C$ identifica a la política francesa. ¿Será casualidad o tendrá que ver con el hecho de que los ideales políticos de la candidata eran totalmente opuestos a los del rotativo? ¿Se nos discutirá, entonces, que los nombres de pila usados con las mujeres políticas tienden a rebajar su imagen pública, sobre todo teniendo en cuenta que este periódico se destacó, además, por el uso de motes o apodos descalificadores para con la aspirante socialista, prácticamente inexistentes en el resto de los periódicos?

Veamos algunos ejemplos de lo apuntado, empezando por un largo texto del mismo $A B C$ en el que reiteradamente se elude nombrar a la política por su apellido, transgrediendo incluso las más elementales reglas de estilo:

[...] que los líderes conservadores interpretan como un «ataque de nervios» del equipo de Ségolène, vapuleado por una ola de sondeos negros [...]. El indicador más alarmante para Ségolène es el descenso por debajo del 30 por ciento en la primera vuelta. A la espera de revelaciones de una u otra especie, la caída brutal e inesperada de la credibilidad de Ségolène atiza la crispación más turbia [...]. La campaña se sume lentamente en su fase más polémica y activa. Ségolène ha entrado dando tropezones.

$A B C, 25$ de enero

Señalaremos, además, otros ejemplos del resto de la prensa analizada, que emplean el nombre de pila, especialmente en los titulares: 
- La tierra de Ségolène, entre campestre y presidencial (La Nación, 29 de abril)

- Simplemente, Ségolène (La Vanguardia, 21 de abril)

- Ségolène también (El Mundo, 21 de enero)

- Las audiencias dan la victoria a Ségolène (El Mundo, 21 de febrero)

Con todo, como ya hemos apuntado, el periódico que se lleva la palma en la familiaridad (sospechosa) con la candidata socialista es, sin duda, $A B C$ :

- ZP, Ségolène, ETA y Boabdil (4 de marzo)

- Ségolène no logra detener su caída con la radicalización de los mensajes (21 de febrero)

- Ségolène no crearía un departamento vasco francés (4 de marzo)

- Ségolène critica el poco apoyo de su partido y pregunta si no confían en ella (14 de marzo)

- Ségolène apela a un Québec libre y provoca la protesta de Canadá (24 de enero)

- Ségolène lanza una batería de medidas sociales para frenar la caída en los sondeos (12 de febrero)

- Ségolène arrincona "La Internacional" y se lanza a entonar "La Marsellesa" (24 de marzo)

- Ségolène quiere proponer una nueva consulta sobre el texto europeo (18 de enero)

Otros periódicos, especialmente El Universal, sin seguir una línea tan marcada, también usan y abusan del nombre de pila al nombrar a la candidata en el interior de sus artículos:

- El primer secretario del Partido Socialista francés y pareja de hecho de Ségolène, François Hollande (El Mundo, 3 de abril)

- Pero Ségolène tiene otros méritos que la han convertido en la mujer con más poder en Francia (El Universal, 16 de abril)

- Es la mejor prueba de que Ségolène logró imponerse a las divisiones internas de su partido y a las intrigas de los viejos líderes (El Universal, 23 de abril)

- "Me encantó la audiencia y me encantó lo que me regaló Ségolène", había dicho en aquella ocasión (La Nación, 10 de febrero)

- Ségolène sigue utilizando una frase para responder a sus críticos (El Universal, 18 de abril)

- El cartel electoral de Ségolène (La Vanguardia, 16 de abril) 
- [...] pero la plataforma de Ségolène no estuvo libre de críticas (El Universal, 12 de febrero)

- Ségolène no está de acuerdo con él (La Vanguardia, 19 de febrero)

- El mitin de ayer en el que Ségolène se mostró muy "solemne" (El Universal, 12 de febrero)

- El otro proyecto lo encabeza Ségolène, y propone una reforma constitucional para impulsar una democracia más participativa [...] Ségolène fue la primera mujer presidenta de una región cuando ganó en 2004 en Poitou-Charentes (El Universal, 24 de abril)

Pero lo más llamativo de este aspecto es que el tratamiento diferenciador por cuestión de sexo se aplica incluso en el interior de la frase, cuando se nombra a un personaje femenino junto a sus congéneres de igual o parecido rango, lo que nos permite hablar ya claramente de discriminación por razón de género o, más exactamente, de sexismo en la prensa escrita. Los ejemplos transcritos a continuación — todos titulares de $A B C$ - no dejan duda al respecto, pues tal cúmulo de despropósitos sexistas no puede deberse más que a una manipulación premeditada:

- Los socialistas franceses exigen que se verifique si Sarkozy mandó investigar al entorno de Ségolène (24 de enero)

- La guerra sucia contra Ségolène y Sarkozy se dispara en "blogs" (27 de enero)

- Ségolène suspende a su portavoz por insultar a su pareja, Hollande (19 de enero)

- François Bayrou asoma ya como alternativa de izquierdas a una Ségolène a la baja (3 de febrero)

- Los "elefantes" acercan a Ségolène al empate técnico. La entrada en campaña de Jospin, Fabius y Strauss-Khan anima a los socialistas clásicos (27 de febrero)

- Ségolène y Sarkozy recurren a sus centristas para frenar a Bayrou (10 de marzo)

- Bayrou sigue sumando apoyos y alcanza por primera vez a Ségolène (12 de marzo)

- Sarkozy conecta mejor que Ségolène con las clases populares (16 de enero)

- Bayrou y Ségolène, empatados (11 de marzo)

- El imparable ascenso de Bayrou amenaza con tumbar a Ségolène en la primera vuelta (9 de marzo) 
Sin embargo, nos equivocaríamos al atribuir tales atrevimientos solo a las diferencias ideológicas entre el periódico $A B C$ y la candidata, pues el rasgo sexista prevalece sobre el ideológico, como demuestran los ejemplos de los restantes rotativos consultados:

- Las fisonomías de Ségolène, Sarkozy, Bové y Chirac disparan los tebeos electorales (El Mundo, 6 de marzo)

- Algunas reconstruyen irónicamente la infancia accidentada de Ségolène y de Sarkozy (El Mundo, 6 de marzo)

- [...] cuando Ségolène derrotó a los candidatos más fuertes de su partido, Laurent Fabius y Dominique Strauss-Khan, se registró un cambio ( $E l$ Universal, 24 de abril)

- [...] había un candidato seguro para la segunda vuelta: Sarkozy. Pero que el otro estaba en duda entre Ségolène y Bayrou (El Universal, 24 de abril)

\subsection{Apelativos, motes, apodos}

Candidata socialista, aspirante al Elíseo, política gala, etc. son términos que se emplearon a menudo para calificar a Ségolène Royal. Hemos encontrado una gran profusión de ellos, a excepción de un ejemplo muy ilustrativo - $\mathrm{y}$ afortunadamente insólito- del diario mexicano, que se resistía a calificarla con la variante de género femenino:

La más reciente fue el domingo pasado por parte de Ségolène Royal, el candidato socialista a la presidencia de Francia

El Universal, 14 de febrero

Por lo demás, y salvo esta poco representativa excepción, los apelativos que se le dirigieron desde los distintos rotativos hispanos no variaban en esencia, al menos en lo que refiere a los que podríamos calificar como "neutros", descriptivos y no valorativos. Sin embargo, no todos los calificativos que acompañaron a la candidata gala en su camino hacia el Elíseo fueron tan asépticos; seguidamente mostraremos algunos mucho menos neutrales. En cualquier caso, no podemos acusar en exclusiva, ni mucho menos, a los rotativos hispanos de la creación de apelativos descalificadores en relación con la aspirante francesa, pues sus propios compatriotas — adversarios políticos o incluso compañeros de partido- lo hicieron antes, desde decir que "Royal es una de las muchas hijas de Mitterrand" (Le Pen) — haciendo, de paso, referencia explícita a la vida privada del antiguo presidente- hasta 
calificarla como "la Madonna de las encuestas" (Le Figaro) y la "gacela", en oposición a "los elefantes" de su partido, o como "la alteza real de Charentes" (otra vez Le Pen).

Otros calificativos se referían a su condición de madre de familia, y no precisamente de forma positiva:

- Royal, que siempre ha explotado su faceta de "supermamá" y que no ha dudado en exhibir sus embarazos y partos en las portadas de la prensa del corazón $(A B C, 3$ de abril)

Con todo, los apodos ridicularizadores preferidos del periódico madrileño —nada afín, como se sabe, a su línea ideológica- son los que la relacionaban directamente con Robespierre o con el presidente Zapatero, quien la apoyó en su campaña:

- El calificativo de "robespierrette", que ridiculiza a Robespierre y a la candidata del PS, confirma una crisis de fondo ("La izquierda cultural critica a "robespierrita»", $A B C, 12$ de febrero)

Hemos de destacar que este periódico prefería, por encima de todos, el apodo de Zapatera, con el que, como se explica en La Vanguardia del 16 de abril, fue bautizada Ségolène Royal tras su espectacular triunfo en las elecciones regionales de 2004. Dicho apodo permitió a $A B C$, que lo repitió insistentemente a lo largo de los meses de campaña, ridiculizar mediante un solo apelativo a dos de sus demonios mediáticos:

- Ségolène Royal, que comenzó a ser tratada cariñosamente de "Zapatera", antes de convertirse en "Robespierrette" ( $A B C, 12$ de febrero)

- La candidata socialista a la presidencia francesa, Ségolène Royal, ha presentado un argumento más que da la razón a aquellos que la llaman la "Zapatera", algo que para ella es "un cumplido" ( $A B C, 26$ de febrero)

Incluso lo destacó en titulares: Royal, la "Zapatera" francesa (23 de enero), Zapatero y zapatera (29 de enero), La Zapatera (14 de febrero), y Zapatero apoyará a 'La Zapatera' (3 de abril). Insistieron en ello diversos artículos de opinión:

- Allí todavía no hay listas de cremallera, al menos hasta que Ségolène Royal, alias Zapatera, gane las elecciones presidenciales $(A B C, 20$ de enero)

- Ségolène Royal ha perdido pie en las encuestas y su perfil es hoy, más que nunca, el de la auténtica «Zapatera».... Para ser la Zapatera perfecta sólo se le olvidó prohibir el tabaco y el vino $(A B C, 14$ de febrero) 
Por otra parte, y como era de prever, resultaba inevitable, a lo largo de la campaña, que los calificativos más o menos ingeniosos que acompañaban al nombre de la aspirante francesa hicieran referencia, otra vez, a su condición femenina:

- La candidata del Partido Socialista, Ségolène Royal, otrora la reina indiscutida de todas las encuestas (El Universal, 19 de abril)

En esta línea se llevan la palma en cuanto a apodos sexistas los del periódico La Vanguardia. Así, titulares como

- Heroína sin clan, novia de Francia (La Vanguardia, 23 de abril),

- Juana de Arco contra Napoleón (La Vanguardia, 22 de abril),

- El rostro de Marianne (La Vanguardia, 21 de abril)

y subtítulos como "La socialista Ségolène Royal, sacerdotisa de la «democracia participativa»" (La Vanguardia, 19 de abril), dejan bien clara la poca sensibilidad de género del rotativo barcelonés, al supeditar la equidad y la corrección sexista a los banales juegos retóricos de sus corresponsales.

\section{Diminutivos}

Si hemos destacado como característica de género el que a las mujeres políticas se las llamara extensamente en los medios públicos por su nombre de pila, nos queda todavía una vuelta más en la tuerca de los despropósitos sexistas: los diminutivos o nombres abreviados y alterados (Pili, Cuqui, Nena, etc.) que tanto abundan en la vida real entre las mujeres, pero que, al tratarse de una profesional de la política, no hacen sino acentuar ese siempre arrastrado sambenito de la infantilidad, falta de seriedad y frivolidad que recae en las mujeres profesionales, y que se contrapone a la dignidad y empaque con que se trata a sus compañeros varones.

El caso no afecta solamente a la política gala, ni mucho menos. Baste recordar que hemos señalado ya a una política hispana de ideología contraria, Esperanza Aguirre, a la que varios rotativos - entre ellos uno tan poco dado a frivolidades como La Vanguardia (12 de febrero de 2007) - se atreven a llamar, no ya por su nombre de pila, sino con un apelativo familiar que raya la vulgaridad: "En los medios periodísticos más compulsivos está reapareciendo Espe (Esperanza Aguirre) como deseado caballo blanco".

No pensemos que si a Ségolène Royal se la conocía como Ségo antes de las elecciones, después, en caso de haber resultado vencedora, el talante 
de la prensa hubiera cambiado, pues recordemos que Esperanza Aguirre -Espe- era y sigue siendo presidenta de una Comunidad Autónoma, y no se nos ocurre el nombre de ningún otro congénere suyo al que un periódico "serio" se atreviera a llamar con un apelativo parecido (a nos ser que estuvieran poniéndolo en boca de terceras personas).

- Pero Sego es algo más que un catálogo de errores (El Universal, 16 de abril)

- Ségo, nacida en 1953 en Senegal (entonces colonia francesa) (La Nación, 22 de abril)

- Cristina Kirchner elogió en privado la actuación y la carrera política de "Ségo", como apodan cariñosamente a la candidata socialista (La Nación, 27 de enero)

- Sego, como la han bautizado sus admiradores, tampoco es muy convincente (El Universal, 16 de abril)

- No es casualidad tampoco que "Ségo" se haya destacado a lo largo de su campaña por su elegancia y la femineidad de su sonrisa (La Nación, 6 de mayo)

Es cierto que al político galo contrincante también se le conocía en su país por el apodo de Sarko, pero, curiosamente, los periódicos hispanos lo nombran así en muy contadas ocasiones:

- Ayer, Sarko y Ségo comenzaron ya a cargarse todos los referentes y récords electorales (La Vanguardia, 23 de abril)

Pero incluso en esos casos se hace con dos particularidades muy interesantes. Por una parte, es curioso constatar que a Sarkozy generalmente se lo nombra por el apodo solo cuando se contrapone al de su contrincante femenina:

- Barcelona dice 'Ségo' y Madrid, 'Sarko' (La Vanguardia, 24 de abril)

- Ya eliminaron a mi candidato (en las primarias del partido socialista) y ahora tengo que escoger entre Sarko y Sego (El Universal, 4 de febrero)

Por otra parte, y esto es sumamente interesante, en las escasas veces en que se nombra al político galo como "Sarko" suele entrecomillarse el apodo, lo que no ocurre siempre cuando se nombra a su contrincante femenina como Ségo:

- Hay una pregunta que le quita el sueño a "Sarko" y a Ségo (La Nación, 26 de abril) 


\section{Aspecto físico}

Es absolutamente incuestionable que las referencias al aspecto físico son omnipresentes en los artículos de prensa que hablan de las mujeres dedicadas a la política. El aspecto, la indumentaria, las maneras y la apariencia, en general, se tienen mucho más en cuenta al hablar de las mujeres públicas que al hacerlo de los varones. Y es también incuestionable que "ciertas" mujeres resultan más proclives que otras a este tipo de comentarios. Si se alude al físico de Angela Merkel o de Michelle Bachelet, se hace generalmente para resaltar algunos aspectos negativos relativos a su ausencia de coquetería: su aspecto ligeramente masculino, su poca atención a los detalles de la vestimenta o el peinado y, en general, el escaso esfuerzo que demuestran por resaltar los atributos de su feminidad. Paradójicamente, es también el aspecto físico, pero en sentido contrario, lo que destacan negativamente en las mujeres públicas que se esfuerzan en mostrar una imagen femenina, elegante y sofisticada. En resumen, lo que en unas es criticable por ausencia en otras lo es por presencia; el caso es que en unas y en otras la apariencia física tiene un valor desmesurado, muy por encima, naturalmente, del que se concede a sus colegas masculinos.

Ségolène Royal pertenece a ese grupo de políticas de las que resulta muy difícil hablar sin hacer alusión alguna a su apariencia. Comparte cartelera con Hillary Clinton, Cristina Fernández de Kirchner, la reina Rania de Jordania y algunas otras, pero, indiscutiblemente, la socialista francesa ocupa el número uno en este ranking.

Su procedencia geográfica colaboró en gran medida a despertar el imaginario de los periodistas, que la consideraron desde un principio digna representante de la feminidad gala:

- Royal contaba como arma con su novedoso charme y su asamblearismo... (La Vanguardia, 14 de abril)

- [el socialismo] trata de sobrevivir en las próximas elecciones presidenciales, recubriéndose astutamente con la aureola «chic» de Ségolène Royal $(A B C, 21$ de marzo)

- Ségolène Royal para seducir a los electores alcanza cotas de notable refinamiento (La Vanguardia, 16 de abril)

- [...] cuando las bases del partido se inclinaron ante el encanto de Ségolène Royal (El Universal, 19 de abril)

- En Ségolène Royal, además de la feminidad, destacan su regionalismo y sus hondas raíces francesas (La Vanguardia, 21 de abril) 
La opinión pública —-mejor, la opinión publicada—, en su afán por destacar las "cualidades" que adornaban a la candidata gala (¿suponían acaso que eran decisivas a la hora de gobernar un país?), no dudaba en compararla -haciéndole un flaco favor-con las más rutilantes estrellas del universo mediático:

- La candidata del PS ya tuvo éxito en conseguir un triunfo envidiable: sus compatriotas la eligieron la sexta mujer con más sex-appeal, por encima de Kate Moss, Naomi Campbell y Penélope Cruz (El Universal, 16 de abril)

- Ségolène Royal y Sharon Stone tienen varios puntos en común. Ambas son mujeres que en su madurez se expresan de forma contundente. Ambas son seductoras y fotogénicas. El cartel electoral de Ségolène Royal que muestra su rostro en primer plano constituye una fotografía magnífica en blanco y negro obra de Emmanuel Scrocelletti, el fotógrafo de las estrellas, autor de un libro sobre Sharon Stone y habitual entre los bastidores del festival de cine de Cannes (La Vanguardia, 16 de abril)

Resulta casi imposible encontrar otras referencias a su persona más allá de las relativas a su condición de mujer atractiva, que se repite machaconamente junto a su nombre, sea cual sea el periódico al que nos dirijamos:

- Con su encanto y con su estilo desestructurado, la candidata recuperó el fervor juvenil (La Nación, 30 de abril)

- Según esa mujer bella y elegante de 53 años, la política ha cambiado de naturaleza (La Nación, 23 de abril)

- Ségolène Royal, una mujer de 53 años, madre de cuatro hijos y dueña de una sonrisa cautivante (El Universal, 22 de abril)

- Royal, una mujer hermosa de 53 años y madre de cuatro hijos (El Universal, 19 de abril)

- La hermosa y popular candidata del Partido Socialista, Ségolène Royal (El Universal, 15 de enero)

Y así hasta la saciedad, aunque algunos periódicos no puedan evitar deslizar un toque negativo entre tanta alabanza

- [...] su maestría de comunicación; mezcla del encanto de su sonrisa —se rehízo la dentadura- y su elegancia sencilla y de cálculo $(A B C, 3$ de abril)

- Se le acusa de ser pura sonrisa (La Nación, 30 de abril) 
- Es difícil seguir ocultando que detrás de una rutilante fachada hecha a medida [...] ( $A B C, 17$ de febrero)

Como veremos más adelante, la ropa usada por la candidata era examinada desde todos los ángulos posibles (¿hablaba alguien de las corbatas o de las chaquetas de Sarkozy?).

- Con sonrisa permanente, maquillada sutilmente y vestida con su chaqueta blanca fetiche $(A B C, 3$ de abril)

- Casi dos horas después del cierre de los colegios electorales Royal, vestida con un impecable traje de dos piezas de color blanco y luciendo su mejor sonrisa (El Universal, 23 de abril)

- El blanco refleja lo puro y sin mácula, de acuerdo con la imagen que la candidata reivindica implícitamente (La Vanguardia, 16 de abril)

Ni siquiera en el momento final y trascendente de las votaciones quedó fuera del espacio mediático su condición de mujer; así, se recurrió, incluso, a metáforas de claro regusto sexista:

- Había votado poco después de mediodía con un semblante radiante y confiado, vestida de blanco para su gran boda con Francia (La Vanguardia, 23 de abril)

En algunos ejemplos queda especialmente patente la variante discriminativa de género, explicitada al adjudicar epítetos a uno $u$ otro candidato:

- Los franceses quedaron enfrentados ayer a un duelo inédito entre la hermosa candidata socialista de 53 años y el temido político conservador de 52 años (El Universal, 24 de abril)

El lenguaje, siempre transparente, se delata en un artículo de opinión de El Universal del 16 de abril, donde se habla de un "arma secreta" que "embrujó a la nación" ("Sonreír, arma secreta de Royal"). Bajo la apariencia de elogio, el periodista no logra disipar su tendenciosidad de género, que queda manifiesta en el adverbio de su subtítulo:

- Su inteligencia y combate a 'elefantes' del PS también favorecen su éxito.

Siguiendo con las alusiones a su vestimenta, abundan los ejemplos en que se hace referencia a la falda, la prenda femenina por excelencia. Entre ellos podemos aportar algunas frases que merecen pasar al museo de los disparates sexistas: 
- Ségolène Royal, bella, con cuatro hijos y pareja de hecho según la ley, ha realizado su campaña electoral en falda (El Universal, 24 de abril)

¿Importará eso a alguien que no sea el periodista y su sesgada visión?, nos preguntamos. En cualquier caso, este periódico mexicano parece tener una especial fijación en el atuendo que expresa - para el periodista- la esencia de la feminidad, puesto que definía ya a la política gala como "una mujer que pocas veces porta pantalones y prefiere la falda y es la compañera, con hijos, de otro socialista" (7 de marzo). En otro inspirado ejercicio retórico insistía en la identificación entre atuendo y género: "Las faldas turban, al parecer, a los elefantes misóginos" (16 de marzo).

Por lo visto, la prensa escrita no era la única que no olvidaba en ningún momento la condición femenina de la aspirante y sus hábitos vestimentarios; así, tampoco se dejó de lado en el debate televisivo entre los dos candidatos, que generó gran expectación en Francia y fue seguido por millones de espectadores: "el flanco de la mesa ubicado frente a las cámaras estará cubierto para impedir que se vean las piernas", según informó previamente La Nación del 10 de abril.

\section{La condición femenina: el hecho de ser mujer}

Para cualquier lector habitual de periódicos, es evidente que el tratamiento que recibió Ségolène Royal en la prensa escrita dista mucho del que ha recibido, y probablemente recibirá, cualquier candidato masculino en unas elecciones libres del mundo occidental. Y no estamos hablando de las críticas, siempre despiadadas, del sector de la prensa ideológicamente contraria a los distintos candidatos, pues esta no es una prerrogativa femenina, ni mucho menos; al contrario, hablamos de cualquier tipo de prensa, que incluso con la voluntad de elogiar no puede pasar por alto la condición de mujer de la candidata, mujer ante todo, por encima de cualquier otra cualidad o defecto en su faceta profesional de política, faceta que debería ser, en definitiva, la que se juzgara.

No estamos diciendo que a otros políticos hombres no se los haya ridiculizado o elogiado en alguna ocasión a propósito de su aspecto físico, su vestimenta o su comportamiento. Estamos hablando de una cuestión de proporción; estamos destacando, sobre todo, la cantidad y la calidad de estas referencias. La desproporcionada relación entre lo que se dice acerca de 
la vida privada o de la apariencia física de un candidato varón y las innumerables referencias - cotilleos, exactamente- que acompañan y adornan la imagen que los poderes mediáticos dan de las mujeres políticas, se aprecia allá donde se mire, desde los elogios desmesurados -empezando por Zapatero, quien "considera «decisivo» que una mujer pueda ser elegida presidenta de Francia" (La Vanguardia, 16 de abril) — hasta las inevitables y tediosas referencias de género, omnipresentes en los medios de comunicación durante la campaña electoral francesa:

- Royal, la primera mujer con posibilidades reales de llegar al Elíseo (El Universal, 14 de enero)

- La primera mujer con opciones de llegar al Elíseo (La Vanguardia, 19 de abril)

- ¿Una mujer para pilotar Francia? (La Vanguardia, 20 de abril)

- Una mujer tras el Elíseo (La Nación, 22 de abril)

- De derecha, de centro o de izquierda, el futuro jefe de Estado (¿o será una jefa de Estado?) (El Universal, 22 de abril)

- Ségolène Royal, la primera mujer en Francia que, desde ayer, tiene serias posibilidades de ocupar el Elíseo, el emblemático palacio que sirve de sede a los presidentes de Francia (El Universal, 23 de abril) (obsérvese el sutil juego del masculino genérico)

- Por primera vez una mujer, Ségolène Royal, candidata del Partido Socialista, pasa a la segunda vuelta para disputar la presidencia del país ( $E l$ Universal, 24 de abril)

No pensemos, sin embargo, que esta obsesión por destacar la identidad de género es privativa de la prensa hispana. En su propio país, la liberal y progresista Francia, el mismísimo exministro socialista Laurent Fabius, al plantearse la candidatura de Ségolène Royal, se había atrevido a ironizar con un "¿Y quién se ocupará de los niños?", según relata La Nación del 23 de abril. También Sarkozy había frivolizado sobre estos aspectos al ser preguntado sobre la dificultad suplementaria de enfrentarse a una mujer, y responder que, aunque era una dificultad añadida, "también es algo novedoso y añade un cierto picante a esta campaña" (El Mundo). Otro francés, Jean-Pierre Raffarin, decía de ella que "seduce de lejos e irrita de cerca", según $A B C$ del 3 de abril. En este mismo sentido, La Vanguardia del 18 de abril recoge que Le Monde escribía que "un presidente debe «tener cojones»" y "claro - apostillaba el periódico catalán- que Royal no los tiene". Para demostrar que ni las mismas mujeres francesas quedaron libres del influjo 
sexista, leemos en el $A B C$ del 12 de febrero que su compatriota, la cantante Françoise Hardy, declaró al respecto: "La preferiría menos atractiva en el terreno de la imagen y más coherente, menos demagógica en el terreno de las ideas".

Veamos, finalmente, un retazo de un largo artículo de la Tribune de Genève del 20 de abril, reproducido por La Vanguardia y titulado ¿Una mujer para pilotar Francia? en el que queda bien claro que "ser mujer" es la principal y omnipresente cualidad del personaje:

¿Francia va a dar un paso histórico el próximo domingo propulsando una mujer a la segunda vuelta de la elección presidencial? Nada está seguro, ya que los sondeos dan a los cuatro candidatos principales, Sarkozy, Royal, Bayrou y Le Pen, una apretada intención de voto. La única mujer de este cuarteto, Ségolène Royal, puede ser apartada como lo fue Lionel Jospin hace cinco años. ¿Francia no está madura para aceptar ser dirigida por una mujer? Difícil de creer. Aparte de algunos machos primarios, nadie seriamente afirma que una mujer no sea apta para dirigir un país.

Mujer, sexualmente atractiva y madre: he aquí los tres pilares que sustentaban, según la prensa, las cualidades políticas de la candidata a la presidencia en uno de los más importantes países del mundo occidental. Buen ejemplo para el resto del orbe.

Por otra parte, es posible también que la misma Ségolène Royal apoyara este tratamiento, pensando que le convenía no desaprovechar sus recursos, ya sea la elegancia, la belleza o la maternidad. Al menos así se desprende de la frase "Es una madre quien les habla", que al parecer repitió en sus discursos siempre que lo creyó oportuno, y de los posados que protagonizó en la prensa junto con sus hijos, principalmente para revistas como Elle o Paris-Match, destinadas a un público femenino. Aun así, lo que merece la pena destacar es el hecho de que, si esta condición resulta aprovechable desde el punto de vista del marketing electoral, demuestra palpablemente lo que tiene de inédito, de revulsivo social -incluso en una de las sociedades más avanzadas del siglo XXI-, que una mujer atractiva, elegante y madre de cuatro hijos, ose competir con un varón en la palestra política.

Ante las abundantes referencias a su condición femenina, la propia Ségolène Royal denunciaba públicamente la poca consistencia de muchas de las acusaciones de sus detractores: "Si me hacen ese tipo de ataques personales es porque no tienen gran cosa que reprocharme", proclamaba, señalando de paso que a un hombre con su trayectoria — diputada, ministra y presidenta regional- no se le harían este tipo de críticas. 
Esas críticas eran a veces explícitas, pero muchas otras veladas, porque hay palabras - verbos, sustantivos o adjetivos - que, aunque se relacionan directamente con la carrera política, como seducir, manipular, ambición, etc., cuando se emplean de forma desmesurada, en este caso para hacer referencia a las mujeres políticas, deben analizarse en clave cuantitativa:

- Para la candidata socialista, el de ayer fue el punto culminante de sus intentos de seducir al electorado centrista (La Nación, 9 de abril)

- Royal, de 54 años, convirtió su condición de mujer en un arma estratégica (La Nación, 6 de mayo)

- El mérito de Ségolène Royal es que hace apenas un año y medio nadie sabía nada de sus ambiciosos planes (La Vanguardia, 24 de abril)

- Ségolène, que ha explotado a fondo la carta de género y su faceta de madre de familia numerosa, es dueña de un estilo personal y de encanto (La Nación, 22 de abril)

Incluso cuando se pretende elogiar a la política, el tono de los comentarios y algunos elementos léxicos valorativos - subjetivemas, en la terminología de Kebrat-Orechionni- no nos dejan olvidar que nos encontramos ante un político que, ante todo y por encima de todo, es una mujer:

- "Muy serena y sólida", Royal se declaró "preparada" y "la única que puede hacer el cambio profundo que necesita Francia" (El Mundo, 21 de febrero)

Y, lo que es peor, en muchas ocasiones la variante de género aparece claramente reflejada en ciertas sutilezas léxicas muy reveladoras:

- La candidata socialista respondió con soltura a las preguntas y a veces hasta se mostró combativa (El Mundo, 21 de febrero)

\section{Condición civil: el papel de hija, compañera (o esposa) y madre}

En el caso de Ségolène Royal se destacó hasta la saciedad en toda la prensa hispana - y suponemos que mundial— las especiales características de su estado civil, algunas veces de manera harto despectiva, ya que no solo se volvía a señalar en una mujer su condición de "hija", sino que se la llegó a etiquetar, increíblemente, como "madre soltera", con las connotaciones que el término (que, además, no está correctamente usado) conlleva: 
- Ségolène Royal, hija de un oficial del ejército y madre soltera de 53 años (El Universal, 23 de abril)

- La historia de Ségolène Royal, hija de un severo teniente coronel del ejército (La Vanguardia, 15 de abril)

- Ségolène Royal, hija de un general, pareja (sin las bendiciones) de Hollande, primer secretario del Partido Socialista (El Universal, 27 de febrero)

- El primer secretario del Partido Socialista francés y pareja de hecho de Ségolène, François Hollande (El Mundo, 3 de abril)

Por otra parte, se insistía en todo momento en destacar como su papel principal en la vida el de ser madre de cuatro hijos:

- Ségolène Royal, una mujer de 53 años, madre de cuatro hijos y dueña de una sonrisa cautivante (El Universal, 22 de abril)

- Royal, una mujer hermosa de 53 años y madre de cuatro hijos (El Universal, 19 de abril)

Tampoco su pareja, como hemos visto, se libró de las continuas referencias a su especial relación. Así, la prensa se refirió en todo momento al político François Hollande como "primer secretario del PS y padre de los hijos de Ségolène" ( $A B C, 16$ de enero), para dejar bien claro su condición de padres, aun sin la existencia de lazos legales entre ambos:

- Royal obtuvo, como lo recordó ayer su compañero sentimental y padre de sus cuatro hijos, más votos de los que obtuvo Mitterrand en 1981 (El Universal, 23 de abril)

Sin olvidar, por supuesto, de mencionar su especial condición civil, no fuera el caso de que los lectores lo hubieran olvidado, optando en ocasiones por un eufemismo innecesario y ambiguo:

- François Hollande, primer secretario del Partido Socialista y compañero de la candidata de su partido al Elíseo, Ségolène Royal (El Mundo, 11 de febrero)

O recurriendo incluso a la más falaz de las cursilerías (sobre todo teniendo en cuenta el desenlace final de la pareja):

- [...] a la sazón fiel compañera del primer secretario del PS, el sacrificado, consensual y gris François Hollande (La Vanguardia, 23 de abril) 


\section{Conclusiones}

Para terminar esta ya demasiado prolija exposición, no nos queda sino repetir esa vieja máxima que nos permite detectar el sexismo en el seno de una sociedad: Será sexista todo aquello (texto, situación o imagen) que no resista la comparación del contraejemplo. Es decir, dado que los sexos están más o menos equitativamente repartidos en la naturaleza humana, sabremos que existe peligro de sexismo ante una imagen (el Congreso de los Diputados, por ejemplo), un texto o una situación vivida, cuando no resista el contraejemplo, en el que se alterase el orden de los sexos (en ambos sentidos, porque también es sexista la composición de algunas reuniones en que prevalece el sexo femenino, por ejemplo en las reuniones de padres de alumnos o en los mercados municipales, aunque muchas veces la escasa presencia masculina es deseada y asumida voluntariamente).

Volviendo a nuestro estudio, será sexista el contenido de un periódico en el que no se resalte todo lo que hemos visto cuando se trate de un candidato masculino. Porque ipodemos imaginar a periodistas de diarios de prestigio como los analizados elogiando sistemáticamente la belleza de algún candidato (que los hay, también, susceptibles de ser elogiados por su físico) y repasando día a día su atuendo? ¿Haría continuas referencias a su condición de padre (preguntándose cómo concilia su paternidad con la política) y se referiría constantemente a la ausencia de vínculos legales con su pareja? ¿Nos imaginamos la posibilidad de que un candidato presidencial (dos veces ministro) fuera nombrado machaconamente por su nombre de pila, obviando el apellido, frente a sus compañeros o adversarios políticos, nombrados con todo detalle?

No, de buen seguro. Luego la conclusión es obvia.

\section{Bibliografía}

1. Bach, M. et al. (2000). El sexo de la noticia. Reflexiones sobre el género en la información y recomendaciones de estilo, Icaria, Bar

2. Forgas Berdet, E. (2010). Cortesía y/o descortesía en la prensa hispana: la imagen de las políticas en los medios de comunicación. En Orletti, F., Mariottini, L. (eds.) (Des)cortesía en español. Espacios teóricos y metodológicos para su estudio, Roma: Roma Tre/Edice.

3. Kerbrat-Orecchioni, C. (1986). La enunciación. De de la subjetividad en el lenguaje, Buenos Aires: Hachette. 